\title{
Three-Dimensional Anisotropy Contrast (3DAC) Magnetic Resonance Imaging of the Human Brain: Application to Assess Wallerian Degeneration
}

\author{
Hironaka Igarashi, Yasuo Katayama, Toshikazu Tsuganezawa, \\ Manabu Yamamuro, Akiro Terashi and Chojin Owan*
}

Three-dimensional anisotropy contrast (3DAC) magnetic resonance imaging is a new algorithm for the treatment of apparent diffusion tensor using the three primary colors. To determine if 3DAC has a clinical application for human brain, six normal volunteers and twenty patients with supratentorial cerebrovascular accidents were examined using clinical magnetic resonance imaging (MRI), and the changes in the 3DAC images associated with Wallerian degeneration of the pyramidal tract were evaluated. The 3DAC images exhibited impressive anatomical resolution. In all chronic stage patients with hemiparesis, the colors in the pyramidal tract were faded. Patients examined during the acute stage who later recovered from hemiparesis had no visible changes of the 3DAC image, whereas patients who recovered poorly showed distinct color fading in the pyramidal tract within 14 days following stroke. In conclusion, very fine anatomical structures are visible on 3DAC images, and it can be used as a diagnostic tool for the human brain.

(Internal Medicine 37: 662-668, 1998)

Key words: diffusion, cerebrovascular disease, pyramidal tract

\section{Introduction}

Diffusion weighted images, using changes of the apparent diffusion coefficient (ADC), are now being used for the clinical evaluation of central nervous system (CNS) disease. Water motion in neurons is restricted or hindered by axons and the myelin sheath (1-4) causing signal attenuation of diffusion weighted images depending on the direction of the diffusion gradient pulse applied. It is this anisotropic nature of the water in the central nervous system that can be used to evaluate neuronal fibers. The strict evaluation of the nature of diffusion anisotropy requires diffusion tensor analysis (5), producing mathematically oriented tensor images and/or anisotropy images (6-9). These images, however, do not clearly depict nerve fiber orientation making them unsuitable for clinical use.

Nakada et al (10) recently introduced a new algorithm for the preparation of apparent diffusion tensor, three dimensional anisotropy contrast magnetic resonance imaging (3DAC imaging). The images produced are color pictures allowing the unprecedented opportunity for visualizing the anatomical details of the CNS (10-12) that can be easily applied to the clinical magnetic resonance imaging (MRI) system.

In the present study, we evaluated the clinical usefulness of this method using a conventional MRI system. We focused on the morphology of the human pyramidal tract in both normal volunteers and patients with hemiparesis following a cerebrovascular accident. Additionally we evaluated whether this method can be used to predict the prognosis of hemiparesis patients.

\section{Methods}

\section{Patients studied}

We studied six normal volunteers (age 58 to 74 , mean age 63.0 years) with no clinical neurological symptoms or abnormal lesions on $\mathrm{T}_{2}$ or $\mathrm{T}_{1}$-weighted images, and twenty patients (age 41 to 95 , mean age 67.3 years) suffering from moderate to severe spastic hemiparesis due to supratentorial cerebrovascular accidents. Two patient groups, chronic $(n=8)$ and acute $(n=12)$ were studied.

In the chronic patient group, the 3DAC images were taken at least 60 days after the onset of stroke. Each of the patients had

From the Second Department of Internal Medicine, *Radiology, Nippon Medical School, Tokyo

Received for publication September 26, 1997; Accepted for publication June 12, 1998

Reprint requests should be addressed to Dr. Hironaka Igarashi, the Second Department of Internal Medicine, Nippon Medical School, 1-1-5 Sendagi, Bunkyoku, Tokyo 113-0021 
moderate to severe spastic hemiparesis. This group was used to determine if wallerian degeneration could be visualized using 3DAC imaging.

The acute patient group had 3DAC images taken within 14 days after the onset of stroke. Motor function was evaluated using the criteria of Brunstrom (13). Each of the patients had a rating of I to IV when the 3DAC images were taken. The acute patients were further divided into two subgroups, good recovery and poor recovery (14) based on the severity of hemiparesis, 90 to 120 days after onset. Patients at Brunstrom stages V and VI were categorized as the "good recovery group" $(\mathrm{n}=5)$, and those rated I to IV made up the "poor recovery group" $(n=7)$. These two subgroups were used to evaluate the usefulness of 3DAC imaging for determining the prognosis of hemiparesis during the acute stage. Patient characteristics are summarized in Table 1.

\section{MR imaging}

MRI measurements were performed on a 1.5 Tesla MR whole body system (Shimadzu Co. Ltd., Kyoto). Initially, fast spin-echo $\mathrm{T}_{2}$-weighted axial images (TR/TE/acquisition $=5,000 /$ $110 / 2$, slice thickness $7 \mathrm{~mm}$, field of view $23 \times 23 \mathrm{~cm}$, matrix $256 \times 256$ ), were taken to determine the optimal position of the image section. Anisotropic diffusion weighted axial images [DWI, gradient strength $=8 \mathrm{mT} / \mathrm{m}$ in each direction, $\mathrm{TR}=$
$1,600-2,500 \mathrm{~ms}$ (cardiac gated, $2-3 \mathrm{RR}$ ), TE $=140 \mathrm{~ms}, \delta=$ $48 \mathrm{~ms}, \Delta=76 \mathrm{~ms}$ ], were then taken sequentially with diffusion sensitizing gradients along each axis (x: right to left, phase encode direction; $\mathrm{y}$ : anterior to posterior, read out direction; $\mathrm{z}$ : orthogonal to the imaging plane, slice selective direction). The pulse sequences used were conventional spin-echo sequences with Stejskal-Tanner type diffusion sensitizing gradients (15). To eliminate phase error due to a patient's motion, the patient's head was placed in a self-made plastic head holder and secured with a plastic band. Total scan-time including localizer imaging took between 25 and 35 minutes depending on the patient's heart rate.

\section{DAC image processing and data analysis}

The three primary colors (red, green, and blue) were assigned to the gray scale of each of the three anisotropic DWI, $x$, $y$, and $\mathrm{z}$, respectively. These three primary color images were then combined pixel by pixel into a single color image using the full visible color spectrum. Finally, combined images were displayed negatively to obtain a one-to-one correlation between red, green, and blue colors and the $\mathrm{x}, \mathrm{y}$, and $\mathrm{z}$ axis, respectively. This imaging process effectively eliminates isotropic components, since the sum of the three primary colors of identical intensity results in a white out.

Additionally, the color frequency (hue) of each pixel reflects

Table 1. Summary of Patients

\begin{tabular}{|c|c|c|c|c|c|}
\hline \multirow[t]{2}{*}{ No. } & \multirow[t]{2}{*}{ Age } & \multirow[t]{2}{*}{ Diagnosis } & \multirow[t]{2}{*}{ Lesion } & \multicolumn{2}{|c|}{ Brunstrom stage } \\
\hline & & & & Lower & Upper \\
\hline \multicolumn{6}{|c|}{ Chronic Stage } \\
\hline 1 & 55 & Infarction & lt. MCA & & \\
\hline 2 & 60 & Infaction & rt. corona radiata & & \\
\hline 3 & 82 & Bleeding & rt. putamen & & \\
\hline 4 & 65 & Infarction & lt. MCA & & \\
\hline 5 & 41 & Bleeding & rt. putamen & & \\
\hline 6 & 76 & Infarction & 1t. corona radiata & & \\
\hline 7 & 69 & Infarction & rt. corona radiata & & \\
\hline 8 & 86 & Infarction & rt. MCA & & \\
\hline \multicolumn{6}{|c|}{ Acute Stage (poor recovery group) } \\
\hline 1 & 95 & Infarction & rt. corona radiata & III & III \\
\hline 2 & 81 & Infarction & rt. striato-capsuler & III & IV \\
\hline 3 & 63 & Infarction & lt. corona radiata & IV & II \\
\hline 4 & 73 & Infarction & lt. internal cupsle & IV & III \\
\hline 5 & 65 & Bleeding & lt. caudate & IV & III \\
\hline \multicolumn{6}{|c|}{ Acute Stage (good recovery group) } \\
\hline 1 & 62 & Infarction & lt. thalmus (lateral) & VI & $\mathrm{V}$ \\
\hline 2 & 52 & Infarction & rt. corona radiata & VI & $\mathrm{V}$ \\
\hline 3 & 67 & Infarction & lt. internal cupsle & $\mathrm{V}$ & VI \\
\hline 4 & 68 & Infarction & rt. corona radiata & VI & VI \\
\hline 5 & 67 & Infarction & lt. corona radiata & VI & $\mathrm{V}$ \\
\hline 6 & 64 & Infarction & lt. thalmus (lateral) & VI & VI \\
\hline 7 & 55 & Bleeding & lt. caudate & VI & VI \\
\hline
\end{tabular}

MCA: middle cerebral artery. 
the direction of anisotropy. In this study, trichromatic coefficients (percent ratio of the contribution of each primary color) (12) were used to assess the anisotropy in the regions of interest. The blue factor of the trichromatic coefficients (b/rgb) was used to compare the pyramidal tract at the upper pons level.

Image synthesis and analysis were done using a work-station (Sparc 20, Sun Co. Ltd.) and IBM compatible personal computer system. The basic theory of this imaging technique has already been described elsewhere (11).

\section{Statistical analysis}

Statistical analysis was done by Student's t-test. Values are expressed as mean \pm S.D. and $\mathrm{p}<0.01$ was considered significant.

\section{Results}

In the 3DAC images, the pyramidal tract nerve fiber bundle, running perpendicular to the slice, exhibited a dominant blue color allowing it to be clearly distinguished from other structures from the medulla oblongata to the corona radiata (Fig. 1). Other nerve fiber bundles (e.g. corpus callosum, optic radiation) were also clearly seen. The 3DAC image exhibited remarkable anatomic resolution, especially at the level of the brainstem, due to the distinctive direction of the nerve fiber bundles (Fig. 2).
In areas histologically rich in cell bodies (ex. thalamus, caudate, cortex) all three coefficients were about 33.3\%. However, regions containing mainly nerve fiber bundles had distinctive color deviations and trichromatic coefficient depending on their direction (Fig. 3).

In the chronic patient group, Wallerian degeneration of the pyramidal tract was clearly seen and the color intensity was reduced (Fig. 4). In two of the cases, changes in the 3DAC images were evident, even though high intensity of the $\mathrm{T}_{2}$ weighted images was not yet obvious. Additionally, the $\mathrm{b} / \mathrm{rgb}$ coefficient was significantly lower $(36.69 \pm 2.21 \%)$ in the chronic stage patients with Wallerian degeneration than in normal volunteers $(43.46 \pm 2.83 \%)$; (Fig. 5).

During the acute stage, there was both fading of the hue and a significant decrease in the $\mathrm{b} / \mathrm{rgb}$ coefficient $(37.02 \pm 1.46 \%)$ in the ipsilateral pyramidal tract of the "poor recovery group" (Fig. 6). Whereas in the "good recovery group" no apparent changes were seen on the 3DAC images and the trichromatic coefficients $(\mathrm{b} / \mathrm{rgb}$ coefficient $=43.85 \pm 4.74 \%)$ were similar to the control group.

\section{Discussion}

Although the 3DAC image is not a true diffusion tensor mapping image, it is a new semi-quantitative contrast image useful for determining axonal direction. For daily clinical use

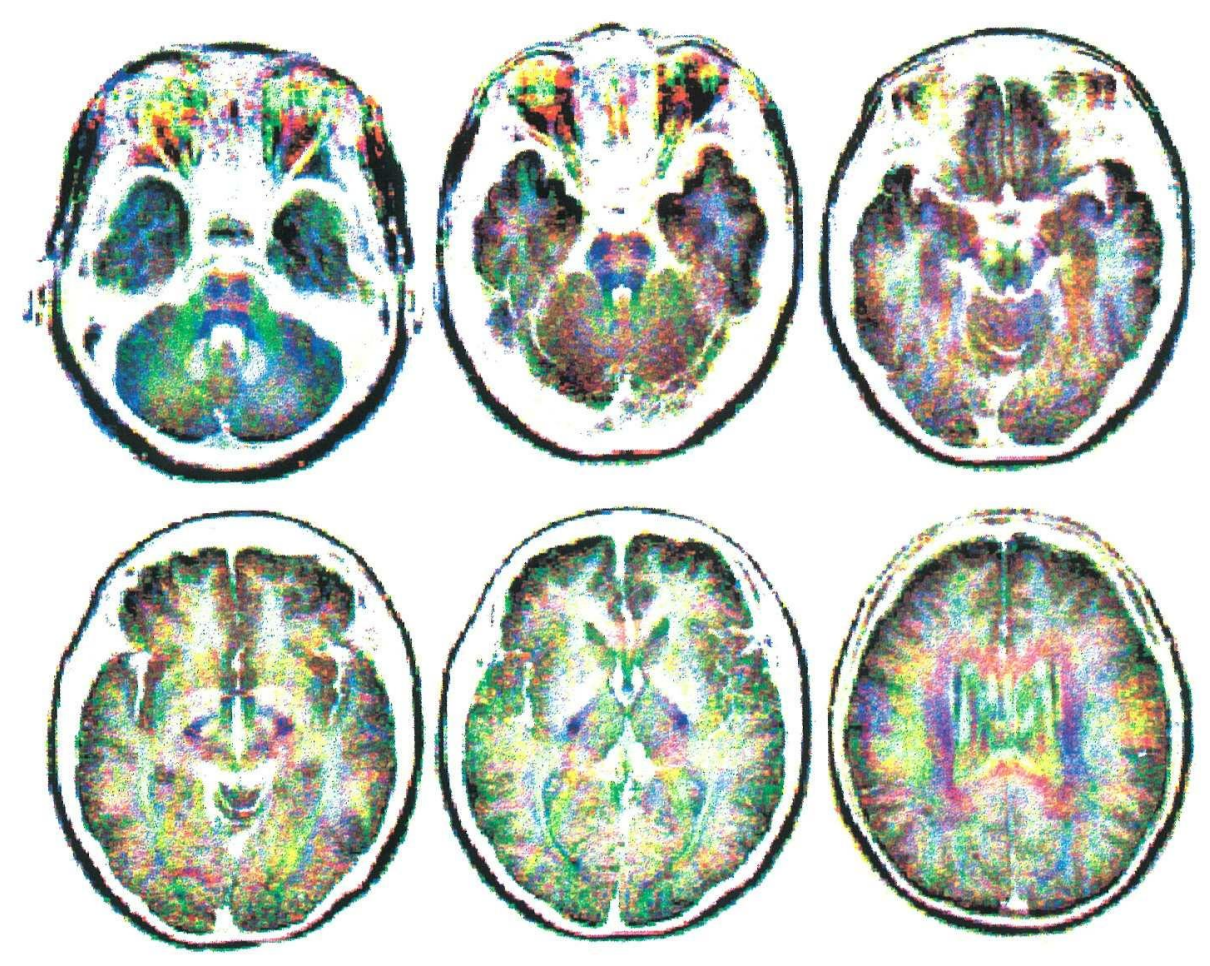

Figure 1. 3DAC images of a normal volunteer obtained from three axial anisotropic diffusion weighted images. The hue of each direction are as follows, right to left: red, anterior to posterior (up and down): green, orthogonal to the imaging plane: blue. The fiber color is dependent on the direction of the image taken. 


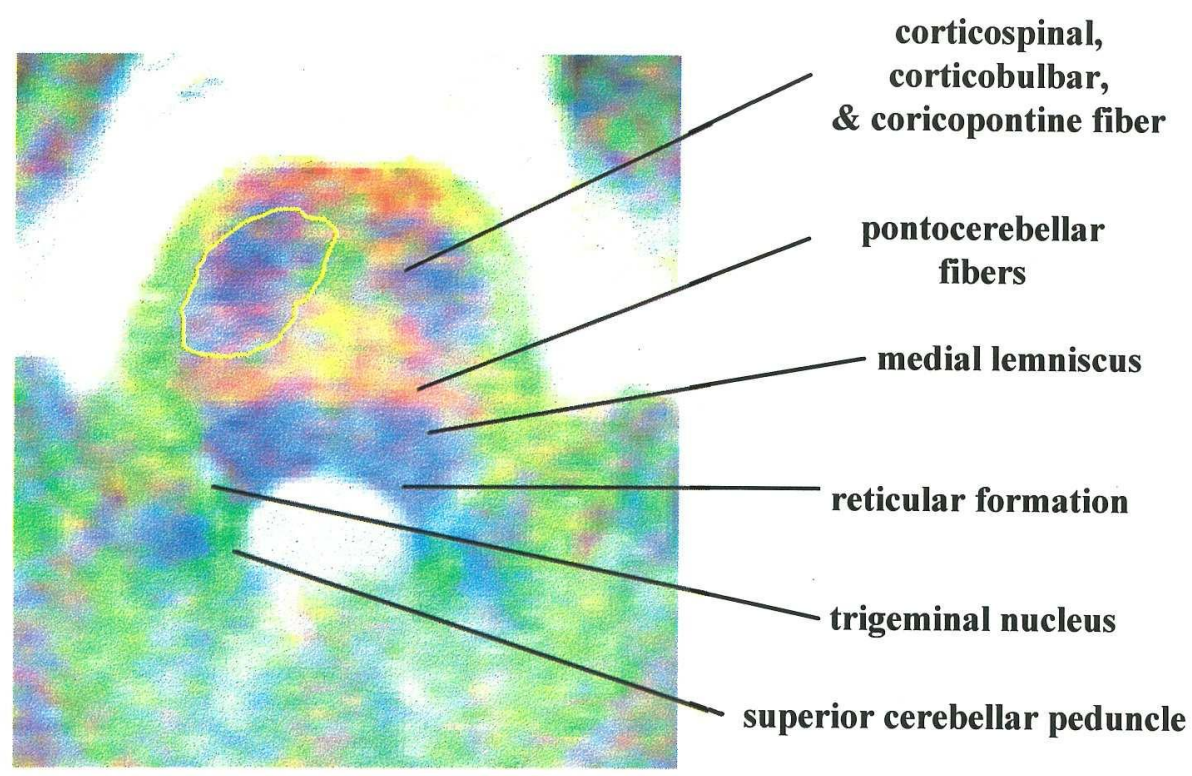

Figure 2. 3DAC image of normal pons. The fine structures of nerve fibers are prominent. The circled area is where the trichromatic coefficients were measured to assess the Wallerian degeneration.

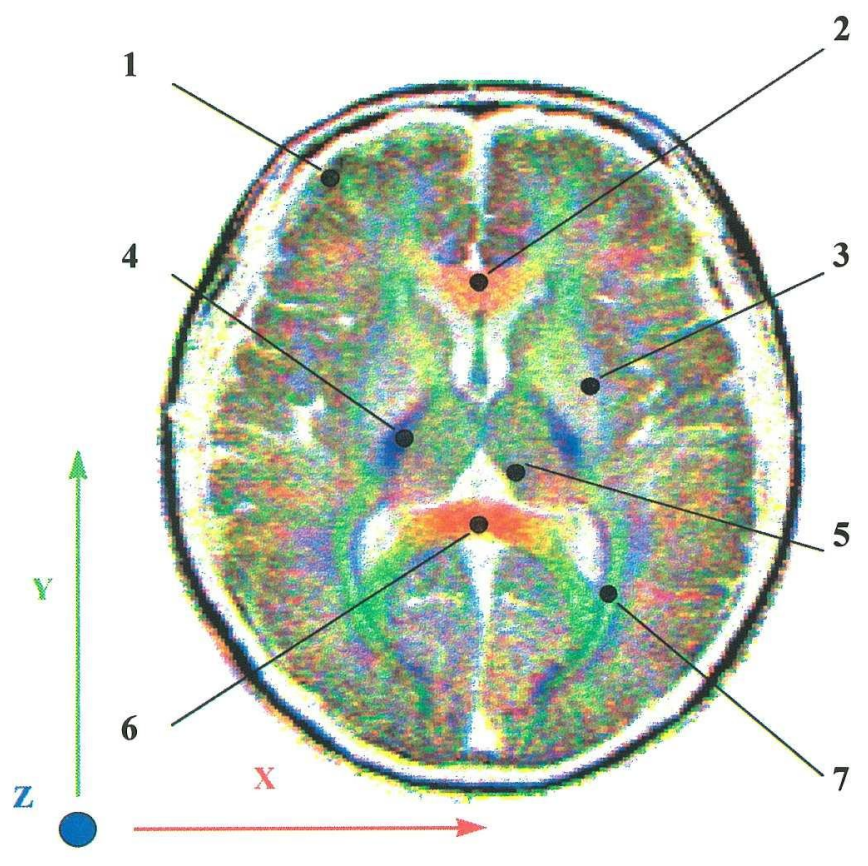

a

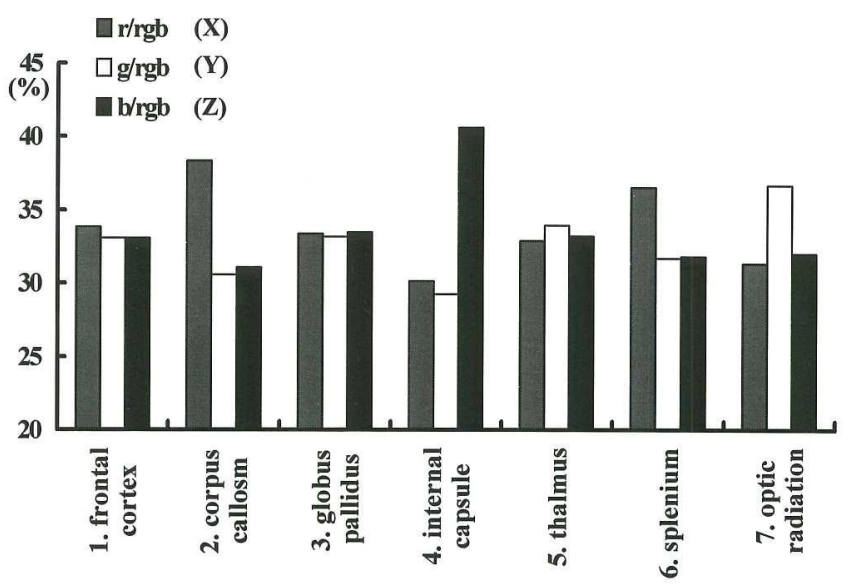

$\mathrm{b}$

Figure 3. Trichromatic ratios of various parts of the brain. Regions which contain mainly nerve fibers (axons) distinctly deviate in the amounts of the three primary colors. In contrast, all three coefficients are nearly $33.3 \%$ in areas histologically rich in cell bodies.

lution capacity providing resolutions similar to those of histological stains. Indeed, Nakada and Matsuzawa (11) stated that 3DAC is the 'stain of in vivo magnetic resonance histology', as the fine anatomical structure of the brain, especially the nerve fiber bundles in the brain stem, are distinctly visible.

In the present study, the 3DAC images were made with a 

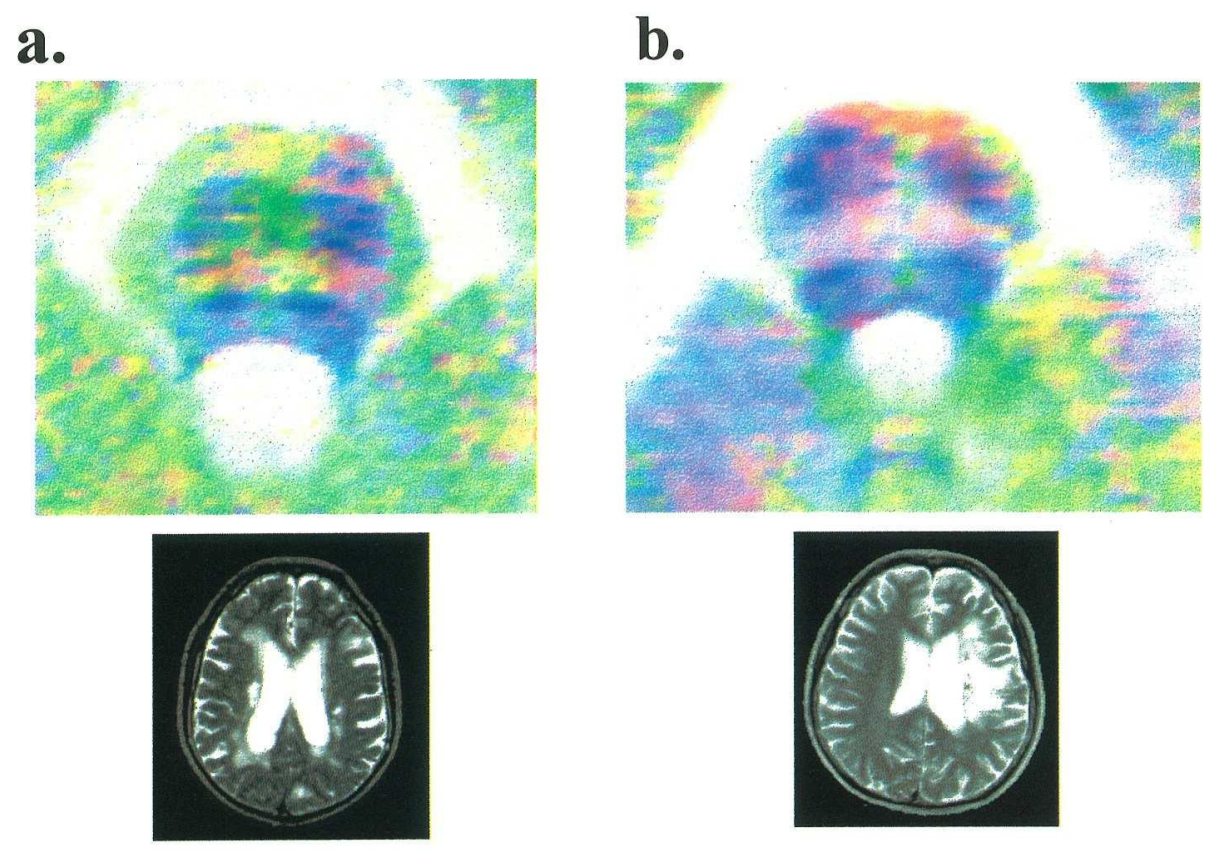

Figure 4. 3DAC images of two chronic patients (a: case 7, b: case 4). Changes in the pyramidal tract are obvious. Especially in case 7 ( 66 days after onset) atrophy of the ipsilateral pyramidal tract is not yet seen, but the fading is clearly noticeable when compared with the contralateral side or with a normal control.

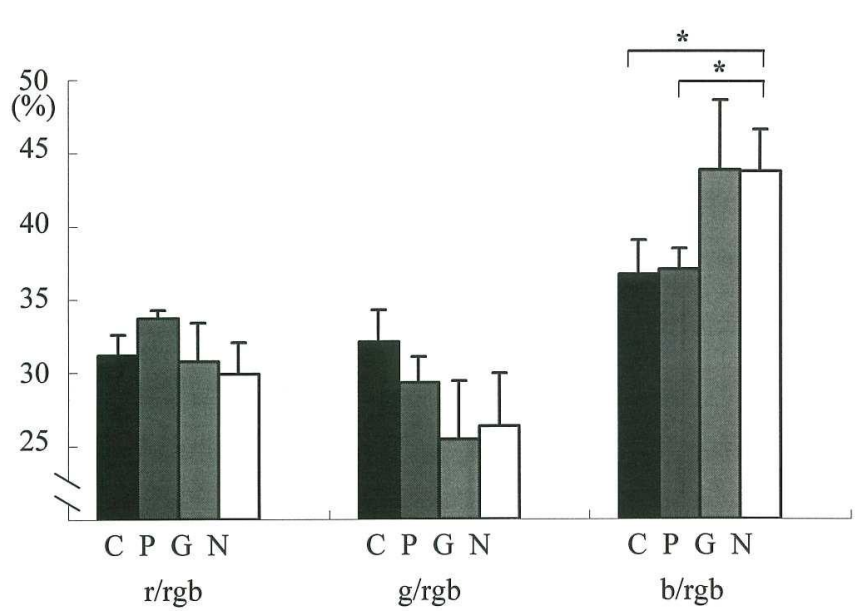

Figure 5. Trichromatic ratios of Wallerian degenerated pyramidal tracts at the level of the upper pons compared with normal controls. *statistically significant $(p<0.01$, student T-test). $(\mathrm{C}$ : chronic group, $\mathrm{P}$ : poor recovery group, G: good recovery group, N: normal control).

relatively low B-value $\left(404.6 \mathrm{sec} / \mathrm{mm}^{2}\right.$ with diffusion time 76 $\mathrm{msec}$, gradient power $8 \mathrm{mT} / \mathrm{m})$. Although Nakada and Matsuzawa (11) suggested that 30 to $40 \mathrm{mT} / \mathrm{m}$ might be needed to make 3DAC images, we were able to construct them from DWI taken with a commercially available MR imager by raising the S/N ratio and using a relatively long diffusion time. The total scan time in our study varied depending on the patient's heart rate, but was less than 20 minutes in most cases. Therefore, 3DAC imaging is applicable in clinical situations.

Since patients are often unable to keep still during the acute stage of stroke, it is difficult to take images using conventional spin-echo pulse sequence. Single shot echo-planner imaging, which eliminates motion artifact, would appear to be a better alternative. It, however, is not suitable because the images are distorted by the susceptibility effect, especially in the infratentorial portion of the central nervous system due to the air-bone spaces, lowering the accuracy in the assessment of Wallerian degeneration in the pyramidal tract.

Using conventional MR imaging $\left(\mathrm{T}_{2} \mathrm{w}\right.$, Proton Density, $\mathrm{T}_{1} \mathrm{w}$ ), wallerian degeneration is typically first revealed two months after the onset of stroke (16-20). Further, some cases show no abnormal signal intensity changes indicating Wallerian degeneration despite symptoms involving upper motor neurons. In the present study, in two of the chronic cases abnormalities of the ipsilateral pyramidal tract were detected using the 3DAC imaging despite the lack of abnormal intensities on $\mathrm{T}_{2} \mathrm{~W}$ image. In the $\mathrm{T}_{2} \mathrm{~W}$ images areas of the myelin sheath with lipid loss appear as high intensity areas, and these changes become evident usually two months after stroke. However, in rat spinal cord with Wallerian degeneration, axon debris disappears within two months after a spinal injury, while the myelin structure is still preserved (21). Since it has been suggested that the diffusion anisotropy (4) and thus 3DAC imaging (10-12) is more closely correlated to the axon rather than the restriction by myelin sheath, the fading pyramidal tract due to wallerian degeneration and the flattening of the trichromatic ratio on the 

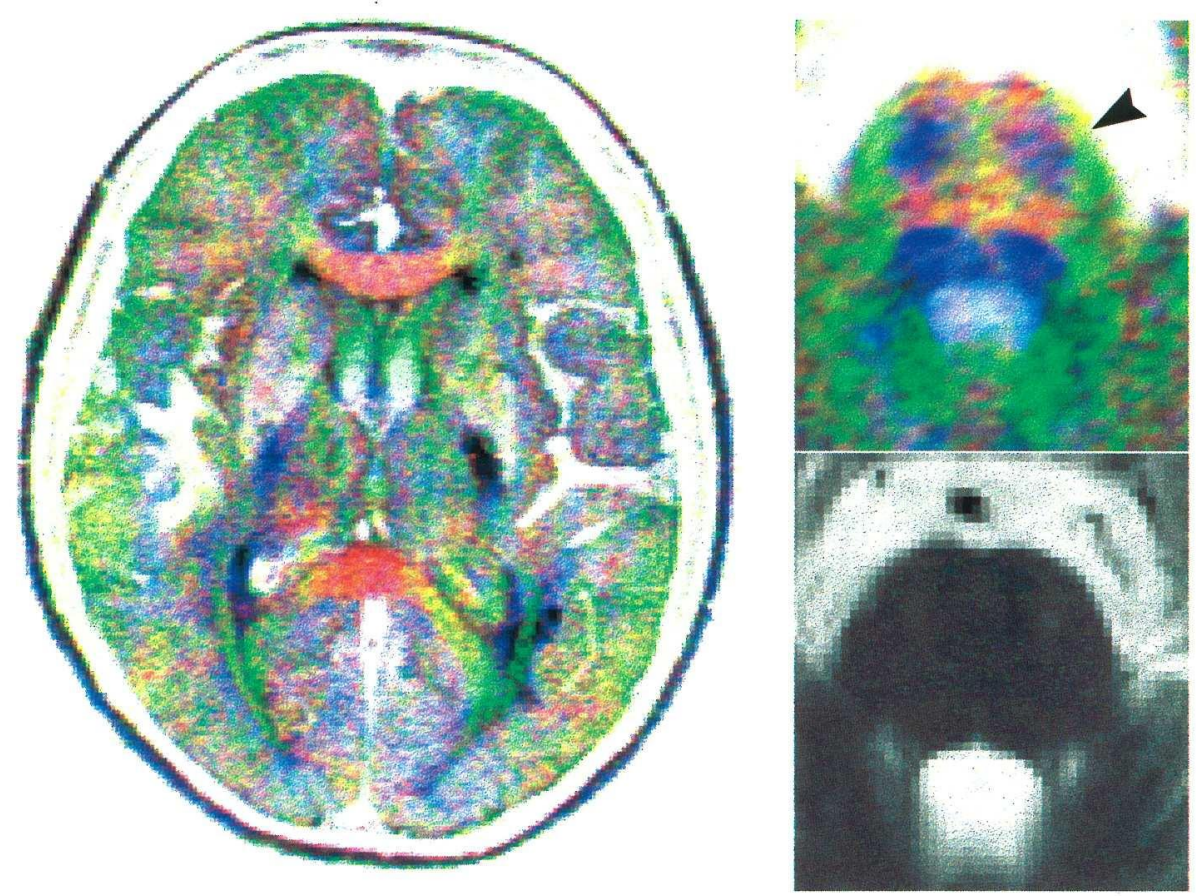

Figure 6. 3DAC image of a patient in the "poor recovery group" (case 4). On the $T_{2^{-}}$ weighted $(5,000 / 110)$ image Wallerian degeneration is not seen. However, on the 3DAC image the changes are clearly seen.

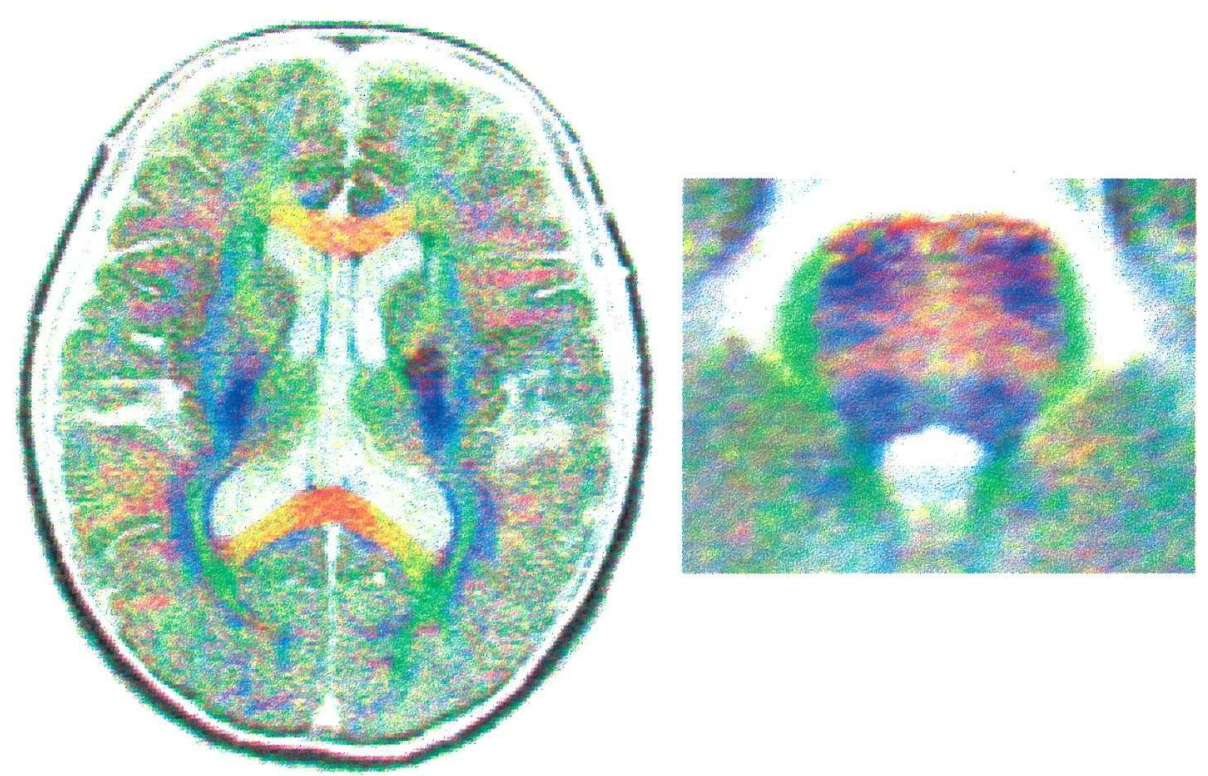

Figure 7. 3DAC images of a patient in the "good recovery group" (case 3). Cerebral infarction was seen in the genu of the internal capsule, but there is no difference in the ipsilateral and contralateral pyramidal tracts. 
3DAC image may be better indicators of Wallerian degeneration than conventional MR images.

During the acute stage, patients who later recovered poorly showed changes in both color intensity and decreases in $\mathrm{b} / \mathrm{rgb}$ coefficients, although morphological changes of the ipsilateral pyramidal tract were not yet prominent. The reason for these changes, although not completely clear, may be attributed to a change in axoplasmic flow. Matsuzawa et al (12), using the rat cardiac arrest model by KCL injection, made chronological 3DAC images of the spinal cord that showed a rapid loss of anisotropy and flattening of the trichromatic coefficients within one hour. He concluded that this phenomenon might be caused by an arrest of axoplasmic flow. From this perspective, it may be possible to assess the degeneration of certain neuronal fiber bundles, making 3DAC imaging a useful diagnostic tool for CNS diseases involving large nerve fiber bundles.

Although the 3DAC images in the present study were done on a conventional clinical MR imager, very fine anatomical structure were visible using a relatively low diffusion gradient power. Therefore, 3DAC images can be used clinically to assess fine anatomical structures and to elucidate alterations of neuronal fiber bundles. Using 3DAC imaging, Wallerian degenerated pyramidal tracts were more visible than with conventional MR images, and the prognosis of motor function could be evaluated using 3DAC imaging during the acute stage of stroke.

In summary, we have shown that $3 \mathrm{DAC}$ can be a very powerful method for analyzing anatomical structures, and moreover it is a sensitive, noninvasive tool for the detection of pathological changes in the human brain.

Acknowledgements: We thank Mrs. Ann Muramatsu for preparation of the manuscript.

Part of this study was presented at the International Symposium on Brain Mapping Oiso '96 (September 13-15th, Oiso, Japan) and the 94th annual meeting of the Japanese Society of Internal Medicine (May 22-25th, Osaka, Japan).

\section{References}

1) Moseley ME, Cohen Y, Kucharczyk J, Mintrovitch J, Asagai HS, Wendland MF. Diffusion-weighted MR imaging of anisotropic water diffusion in cat central nervous system. Radiology 176: 439, 1990.

2) Le Bihan $D$, Turner R, Douek P. Is water diffusion restricted in human brain white matter? An echo-planner NMR imaging study. Neuroreport 4: 887,1993

3) Wimberger DM, Roberts TP, Barkovich AJ, Prayer LM, Moseley ME, Kucharczyk J. Identification of "premyelination" by diffusion-weighted MRI. J Comput Assist Tomgr 19: 28, 1995.
4) Ono J, Harada K, Takahashi M, et al. Differentiation between dysmyelination and demyelination using magnetic resonance diffusional anisotropy. Brain Res 671: 141, 1995.

5) Basser PJ, Mattiello JM, Le Bihan D. Estimation of the effective selfdiffusion tensor from the NMR spin-echo. J Magn Reson B 103: 247, 1994.

6) Coremans J, Luypaert R, Verhelle F, Stadnik T, Oseaux M. A method for myelin fiber orientation mapping using diffusion-weighted MR images. Magn Reson Imaging 12: 443, 1994.

7) van Gelderen P, de Vleeschouwer MH, DesPres D, Pekar J, van Zijl PC, Moonen CT. Water diffusion and acute stroke. Magn Reson Med 31: 154, 1994.

8) Basser PJ, Mattiello J, Le Bihan D. Anisotropic diffusion: MR diffusion tensor imaging. in: Diffusion and Perfusion Magnetic Resonance Imaging, Le Bihan D, Ed. Raven Press, New York, 1995, p. 140.

9) Pierpaoli C, Jezzard P, Basser PJ, Barnett A, Di Chiro G. Diffusion tensor MR imaging of the human brain. Radiology 210: 637, 1996.

10) Nakada $T$, Matsuzawa $H$, Kwee L. Magnetic resonance axonography of the rat spinal cord. Neuroreport 5: 2053, 1994.

11) Nakada $T$, Matsuzawa $H$. Three-dimensional anisotropy contrast magnetic resonance imaging of the rat nervous system: MR axonography. Neurosci Res 22: 389, 1995 (published erratum appears in Neurosci Res 23: 229, 1995).

12) Matsuzawa H, Kwee IL, Nakada T. Magnetic resonance axonography of the rat spinal cord: postmortem effects. J Neurosurg 83: 1023, 1995.

13) Brunstrom S. Movement therapy in hemiplegia. in A Neurophysiological Approach. Harper and Row, New York, 1970.

14) Waragai $M$, Iwabuchi $S$. Wallerian degeneration of the corticodescending tract in the cerebral peduncle following a supratentorial cerebrovascular lesion detected by MRI - The relationship between Wallerian degeneration at the center of the cerebral peduncle and functional recovery of paresis -. Rinsho Shinkei (Clin Neurol) 33: 1146, 1993 (Abstract in English).

15) Stejskal E, Tanner J. Spin diffusion measurements: spin echoes in the presence of time-dependent field gradient. J Chem Physics 42: 288, 1965.

16) Matsumura $Y$, Fukuda $T$, Inoue $Y$, et al. MR images of wallerian degeneration - relation between the time and MR findings of wallerian degeneration. Nippon Acta Radiologica 49: 1168, 1989 (Abstract in English).

17) Kuhn MJ, Mikulis DJ, Ayoub DM, Kosofsky BE, Davis KR, Taversa JM. Wallerian degeneration after cerebral infarction: Evaluation with sequential MR imaging. Radiology 172: 179, 1989.

18) Inoue $Y$, Matsumura $Y$, Fukada $T$, et al. $M R$ imaging of wallerian degeneration in the brainstem: Temporal relationships. AJNR 11: 897, 1990.

19) Pujol J, Martì-Vilalta JL, Junquè $C$, Vendrell $P$, Fernández J, Capdevila A, Wallerian degeneration of the pyramidal tract in capsular infarction studied by magnetic resonance imaging. Stroke 21: 404, 1990.

20) Orita $T$, Tsurutani $T$, Izumihara $K$, Kajiwara $K$, Early, evolving wallerian degeneration of the pyramidal tract in cerebrovascular diseases: MR study. J Comput Assist Tomogr 18: 943, 1994.

21) Lampert PW, Cressman MR. Fine-structural changes of myelin sheaths after axonal degeneration in the spinal cord of rats. Am J Pathol 49: 1139,1966 